\title{
CULTIVAR CONOCIMIENTOS, COSECHAR OTRA EDUCACIÓN EXPERIENCIAS DESDE LA PERSPECTIVA INDÍGENA
}

\section{Sebastián Levalle}

\section{Resumen}

Desde mediados del siglo pasado, algunas organizaciones indígenas latinoamericanas avanzaron en la construcción de experiencias de educación propia. Con foco en la praxis educativa del Consejo Regional Indígena del Cauca, en este trabajo analizo los procesos de investigación indígena que favorecen la construcción de currículos y prácticas interculturales. Me interesa relevar tres cuestiones centrales: a) cómo se ha producido la articulación entre educación propia e investigación, b) el modo en el que esta articulación obliga a reconceptualizar la labor de investigar y c) los elementos que ofrece para profundizar la construcción de praxis socioeducativas alternativas.

Palabras clave: educación bilingüe intercultural, investigación indígena, movimiento indígena, movimientos sociales.

\section{Abstract}

Cultivate knowledge, reap other education. Experiences from the indigenous perspective

Since the middle of the last century, some Latin American indigenous organizations have made progress in building their own education experiences. Focused on the educational praxis of the Regional Indigenous Council of Cauca, in this work I analyze the indigenous research processes that are necessary to build intercultural curricula and intercultural practices. I am interested in releasing three central questions: a) how the articulation between own education and research has been produced, b] the way in which this articulation forces to reconceptualize the work of investigating, and c) the elements that it offers to deepen the construction of alternative socio-educational praxis.

Keywords: intercultural bilingual education, indigenous research, indigenous movement, social movements.

\footnotetext{
Sebastián Levalle: Magíster en Estudios Sociales Latinoamericanos y licenciado en Sociología por la Universidad de Buenos Aires (UBA). Becario doctoral del Consejo Nacional de Investigaciones Científicas y Técnicas (CONICET). Integrante del Grupo de Estudios de Sociología Histórica de América Latina (GESHAL) y el Grupo de Estudios de Memorias Alterizadas y Subordinadas (GEMAS) en la UBA. Docente de grado en la UBA y en la Universidad Nacional de José C. Paz. Ha dictado cursos de posgrado en la Universidad Nacional Arturo Jauretche. ORCID iD: 0000-0002-7860-5603

Email: slevalle@yahoo.com.ar
} 


\section{Introducción ${ }^{1}$}

Las organizaciones indígenas latinoamericanas han denunciado reiteradamente que los proyectos educativos oficiales, aquellos promovidos por el Estado o por las iglesias, violentaron sus formas de vida y negaron sus conocimientos. Las escuelas erigieron una frontera entre "lo indígena", que confinaron al pasado, y el presente, que ellas vinieron a hacer posible bajo el signo del desarrollo, el Espíritu Santo y los colores de la nación. En su afán asimilacionista, la educación oficial - incluso allí donde se impartió en formatos de educación bilingüe- promovió la integración al mundo mestizo, la castellanización y la migración. ${ }^{2}$

Para enfrentar la violencia de este modelo educativo, algunas organizaciones indígenas latinoamericanas han avanzado en el desarrollo de sus propias experiencias de educación. En ciertos casos, como en Colombia (Programa de Educación Bilingüe Intercultural, 2004) o en Ecuador (Vélez, 2008), estas experiencias han sido reconocidas, e incluso impulsadas, desde el Estado. Sin embargo, ocasionalmente, los modelos pedagógicos que animan estas iniciativas no presentan grandes diferencias respecto de los formatos más tradicionales u oficiales.

1 El presente trabajo adelanta elementos de mi tesis de doctorado en Antropología, bajo la dirección de las doctoras Verónica Giordano y Mariela Eva Rodríguez, financiado gracias a una beca doctoral del CONICET de Argentina. Quiero agradecer a los investigadores nasa por compartir su trabajo conmigo y a Alfredo Falero por convocarme al Seminario Movimientos Sociales, Praxis Pedagógicas y Educación, realizado en agosto de 2018 en la Facultad de Ciencias Sociales de la Universidad de la República, espacio en el que surgió la motivación para el dossier del cual forma parte este artículo. Agradezco también a los evaluadores anónimos por las sugerencias brindadas para mejorar este trabajo.

2 Quizás el antecedente más importante en esta materia sea la Declaración de Barbados II, de 1977, en la que un conjunto de líderes indígenas de doce países latinoamericanos denunció que el sistema educativo formal contribuía a los fines de la "dominación cultural". En aquel documento se afirmaba: "La dominación cultural puede considerarse realizada cuando en la mentalidad del indio se ha establecido que la cultura occidental o del dominador es la única y el nivel más alto del desarrollo, en tanto que la cultura propia no es cultura, sino el nivel más bajo de atraso que debe superarse; esto trae como consecuencia la separación por medio de vías educativas de los individuos integrantes de nuestro pueblo" (Bonfil Batalla et al., 1977, p. 111). La crítica al sistema educativo formal ha continuado en múltiples documentos de las organizaciones indígenas del subcontinente (ver, entre otros, Organización Nacional Indígena de Colombia, 2010 [1981]; Primer Encuentro Continental de Pueblos Indígenas, 1990). 
Integrantes del pueblo nasa de Colombia me comentaban que en sus procesos educativos estaban usando su propia lengua "pero para blanquearnos cada vez más" (Ramos, I., comunicación personal, 13 de febrero de 2013). Frases similares he escuchado en Quito, en boca de militantes de la Confederación de Nacionalidades Indígenas del Ecuador (CONAIE), respecto del sistema de educación que hasta el gobierno de Rafael Correa cogestionaban las organizaciones indígenas y el Estado por medio de la Dirección de Educación Intercultural Bilingüe (Morocho, M. y Montaluisa, L., comunicación personal, 17 de octubre del 2009). Tal como solía afirmar Julián Taish Maanchi, profesor de Epistemología en un programa de formación de maestros indígenas de la selva peruana, los intelectuales indígenas suelen estar preocupados, porque los conocimientos cosmogónicos de sus pueblos, sus lenguas y sus prácticas espirituales no logran ganar un lugar central en sus propias prácticas educativas (Taish Maanchi, J., comunicación personal, 13 de agosto de 2009).

Para evitar reproducir las prácticas educativas que tradicionalmente han impartido el Estado y las iglesias en sus territorios, algunas organizaciones indígenas del continente adoptaron la noción de interculturalidad y la opusieron a la de multiculturalismo, de uso más frecuente en las políticas indigenistas de educación bilingüe. El multiculturalismo y la interculturalidad aparecen asociados a una serie de significados en constante redefinición que remiten a una variedad de posiciones dinámicas y ocasionalmente contradictorias. Antes que un atributo de la realidad, estas nociones conforman construcciones históricas, sociales y académicas en constante disputa y negociación (Briones et al., 2006). En la fase actual del capitalismo, la lucha por la hegemonía se despliega, en parte, bajo estas categorías, verdaderos "significantes vacíos" (Laclau, 1996) que adquieren su sentido en los usos históricos que de ellos hacen los actores sociales.

Cuando las organizaciones indígenas del espacio andino apelan a estos conceptos, se ponen en juego los significados que adquirió históricamente la diversidad cultural. En este punto es posible identificar dos posiciones opuestas. Por un lado, la tradición del multiculturalismo anglosajón (Kymlicka, 1996; Taylor, 1999) sostiene la idea de un pluralismo integrador que concibe a la diferencia cultural como un atributo no relacional de los colectivos culturales, justificando las relaciones de poder vigentes. Por el otro, se encuentran las posturas que entienden a la cultura como una arena de lucha (Williams, 1988) y a la diversidad como una forma de desigualdad que exige denunciar el carácter conflictivo de las relaciones culturales y deconstruir el entramado de relaciones de poder que subyacen en el diálogo entre las culturas (Alonso y Díaz, 2004; García Canclini, 2004). En esta línea, María Laura Diez sostiene que las luchas indígenas han configurado un "paradigma intercultural" dentro del cual el concepto de interculturalidad 
es concebido como un "proyecto político, social y epistemológico" (Diez, 2004, p. 197). ${ }^{3}$

El carácter "propio/popular/alternativo" de los procesos educativos de índole intercultural no se agota en la incorporación de problemáticas sociopolíticas, al estilo de los "temas generadores" que Paulo Freire (1970) propuso en Brasil y en Chile. No se trata únicamente de politizar contenidos curriculares y de fomentar el pensamiento crítico. Tampoco alcanza con someter a debate los formatos pedagógicos tradicionales a favor de una perspectiva dialógica. Todo esto es fundamental para construir una educación crítica, e incluso, muchas veces, permanece como tarea pendiente en los procesos de educación que llevan adelante varias organizaciones indígenas. Sin embargo, si estas experiencias se circunscribieran a tales cometidos, continuarían ejerciendo aquella violencia contra sus culturas y sus conocimientos.

Esto ocurre a menudo y lo más preocupante es que esa empresa colonial se lleva a cabo muchas veces en nombre de la emancipación. Es una paradoja que encuentra su explicación en la naturaleza moderna de nuestras tradiciones críticas (De Sousa Santos, 2010). La modernidad ha concebido al conocimiento científico como el único conocimiento válido. Bajo esta lógica, los conocimientos producidos por los pueblos indígenas fueron asumidos como saberes tradicionales y despojados de su capacidad heurística. Tales conocimientos, se afirma reiteradamente, no son verdadero conocimiento. En esta premisa radica el carácter colonial de la modernidad: los pueblos ignorantes no pueden valerse por sus propios medios, deben ser tutelados (De la Cadena, 2009). Para descolonizar la praxis pedagógica en los pueblos indígenas es necesario ir más allá de nuestras perspectivas críticas modernas.

Hay dos elementos del pensamiento crítico que se pueden identificar rápidamente como imposiciones occidentales sobre las ontologías indígenas: nuestra concepción sobre el tiempo y la división entre naturaleza y cultura. El pensamiento moderno europeo postula una temporalidad histórica unilineal (Quijano, 2000). En este esquema, la civilización industrial aparece hacia el

3 Es importante resaltar nuevamente la importancia de los contextos sociohistóricos. Para ello puede resultar esclarecedor tomar dos casos polares como los de México y Uruguay. En México, lo indígena participa de las narrativas nacionales y la educación que imparte el Estado para estas poblaciones es denominada “educación intercultural”. Existe, incluso, la Coordinación General de Educación Intercultural y Bilingüe dentro de la Secretaría de Educación Pública. En Uruguay, por el contrario, el Estado continúa negando la existencia de poblaciones indígenas y no dispone de una política educativa bilingüe, a excepción de aquellas iniciativas ensayadas recientemente en escuelas de frontera en el norte del país para hablantes de español-portugués (Brovetto, 2010). Esta diferencia explica que en México las comunidades zapatistas del estado de Chiapas, que han avanzado en la construcción de experiencias educativas, no hablen de "educación intercultural", sino de educación "autónoma" o "verdadera" (Baronnet, 2013), mientras que las organizaciones charrúas del Uruguay apelan a la interculturalidad para caracterizar a sus iniciativas de formación no escolar. Los charrúas crearon en 2017 la Escuela Intercultural Charrúa Itinerante, que es el fruto de metodologías innovadoras de investigación con interesantes resonancias respecto de las experiencias que abordaré más adelante (Rodríguez y Michelena, 2018). 
final, como la realización de la historia universal, mientras que los pueblos indígenas son ubicados en el comienzo de la historia, como expresiones de un pasado que debe ser superado. Las opciones históricas para estos pueblos van desde el mestizaje y la colonización hasta el genocidio (Lander, 2000). Los pueblos indígenas latinoamericanos piensan el tiempo de formas diversas, pero rara vez lo hacen bajo un esquema lineal y evolutivo.

El otro punto problemático respecto de nuestras tradiciones de pensamiento crítico se refiere a la división entre la naturaleza y la cultura. La modernidad europea instituyó por primera vez una división esencial entre aquello que concebimos como natural y que no es producto de la acción humana y aquello que puede entenderse como cultural y que pertenece a la sociedad (Latour, 1991). La naturaleza es el campo a ser conocido y dominado por el ser humano mediante el conocimiento científico. La cultura es el campo de la agencia, la morada del sujeto. Solamente los modernos hacemos esta distinción entre las cosas y los signos: ni los chinos, ni los koori de Australia, ni los shuar de la Amazonía, ni los kichwa de los Andes sostienen esta idea (Blaser y De la Cadena, 2009; Descola, 2012). Y, sin embargo, esta distinción nos parece natural, incuestionable. Los pueblos indígenas postulan que todo lo que existe tiene vida y agencia propia. Montañas, peces, rocas, árboles entran en comunicación con los humanos. La vida personal y social depende de la coexistencia armónica con todos los seres que habitan en el territorio. El territorio es un espacio donde se recrean conocimientos ancestrales (Rappaport, 2004).

La promesa de una praxis educativa descolonizadora plantea, entonces, problemas impensados: ¿cómo enseñar historia desde una concepción temporal no lineal?, ¿cómo definir las áreas de estudio sin reproducir la división entre naturaleza y cultura? En definitiva, estas preguntas nos remiten a un interrogante central: ¿es posible construir una propuesta educativa intercultural? Un primer paso que suelen ensayar las organizaciones consiste en cuestionar el pretendido monopolio del saber por parte del docente. Tal empresa crítica no se dirige principalmente al diálogo con el estudiante, sino a incorporar otros sujetos al aula: ancianos, mayores, chamanes, parteras, sobanderos, palabreros, etcétera. De esta forma, se intenta legitimar conocimientos que han sido históricamente intervenidos y silenciados por la persistencia de la colonialidad en nuestros países. ${ }^{4}$

4 Experiencias interesantes en este sentido se pueden encontrar, por ejemplo, en el caso del Programa de Formación de Maestros Bilingües de la Amazonía Peruana (FORMABIAP), una institución gestionada por las organizaciones indígenas de la selva del Perú. En el proceso de formación docente, el programa incorporó la figura de los especialistas indígenas, sabedores que se han formado en sus comunidades mediante la memoria colectiva y el uso de plantas medicinales (Trapnell Forero, 2008). El Programa de Educación Bilingüe Intercultural de los pueblos indígenas del suroccidente colombiano también ha logrado el reconocimiento oficial de sus sabedores dentro del proceso educativo (Comisión Nacional de Trabajo y Concertación de la Educación para los Pueblos Indígenas, 2009). 
Pero allí donde se ha avanzado en la construcción de currículos interculturales ha sido necesario ejercitar algún tipo de investigación. Investigaciones que se orientaron a responder interrogantes como: ¿quiénes somos nosotros?, ¿cuál es nuestra historia?, ¿qué decían nuestros abuelos?, ¿cómo interpretamos el mundo?, ¿existe una matemática propia?, ¿podemos producir conocimiento desde nuestra lengua?

Quisiera detenerme a continuación en algunas reflexiones en torno de estas experiencias que podemos denominar "investigación indígena". Encuentro dos buenos motivos para emprender este recorrido. Por una parte, lo dicho: estas investigaciones les permiten a las organizaciones indígenas problematizar - más precisamente, descolonizar - su práctica educativa. Pero, por otra parte, estos procesos nos interpelan profundamente en nuestra labor académica, porque traen a primer plano dos interrogantes que solemos abandonar a su suerte en los pasillos de nuestras universidades: para quién investigar y para qué investigar. Cuando son los propios indígenas quienes responden esas preguntas y cuando nosotros nos asumimos no solamente como académicos sino también como militantes, emerge un problema inquietante: ¿tenemos algún papel para jugar en esta historia?, ¿es necesaria la academia? Son preguntas inquietantes, porque nos invitan a movilizarnos, a poner en movimiento ideas y prácticas. Estas preguntas tienen una interesante historia en nuestra América, ya se las habían planteado Paulo Freire y Orlando Fals Borda, entre otros, en los años setenta y hoy sirven para que reflexione un conjunto de académicos de las más diversas disciplinas, desde la sociología hasta la lingüística y la arqueología (Levalle, 2014). Si bien me voy a enfocar en experiencias indígenas, la potencia de estos interrogantes puede extenderse al conjunto de experiencias sociopedagógicas transformadoras que se abordan en el dossier del que forma parte este artículo.

\section{Investigación y educación indígena}

Joanne Rappaport, una antropóloga que colabora con el movimiento indígena del sur de Colombia, ubica un clivaje importante para comenzar a indagar en las investigaciones indígenas. Según esta autora, tales investigaciones se distinguen de los procesos de formación de intelectuales nativos dentro de las universidades convencionales, algo habitual en países como Bolivia o Guatemala. Si bien en todos los países de la región el ingreso de indígenas en la educación superior adolece de fuertes restricciones (Mato, 2008), resulta habitual que en los casos en los que los estudiantes logran concluir sus estudios tiendan a distanciarse de sus bases de apoyo. Rappaport (2013) afirma que, si bien es necesario continuar con las políticas de graduación de indígenas, la profesionalización termina reproduciendo la lógica del indio permitido (Hale, 2004), conformando actores indígenas que gozan de derechos limitados para hablar y gobernarse. 
Pero Rappaport señala que en los últimos años algunas organizaciones indígenas están logrando construir propuestas propias de investigación. Sus protagonistas no son intelectuales en el sentido clásico, sino militantes con poco entrenamiento académico formal que orientan su labor de acuerdo con los proyectos políticos de las organizaciones a las que pertenecen. Junto a estos intelectuales indígenas suelen colaborar académicos no indígenas de diversas disciplinas (Rappaport, 2013).

Esta clase de iniciativas está atravesando un proceso de institucionalización en América Latina. Desde fines del siglo XX, con la creación de la Universidad de las Regiones Autónomas de la Costa Caribe Nicaragüense (URACCAN) y varias instituciones afines en otros países, las investigaciones indígenas comenzaron a producirse dentro de lo que Daniel Mato (2009) designa como instituciones interculturales de educación superior. Estas instituciones han sido creadas con el objeto de atender demandas y propuestas de formación de pueblos indígenas y afrodescendientes, y sus programas articulan saberes de varias tradiciones culturales. En el interior de este universo - en el que Mato y sus colaboradores registran veintidós casos en nuestro subcontinente- se distinguen aquellas experiencias creadas por organizaciones indígenas de las que han sido impulsadas por organismos del Estado, universidades convencionales, fundaciones privadas u organismos de cooperación internacional.

Para analizar este asunto con más profundidad voy a detenerme en la experiencia educativa del Consejo Regional Indígena del Cauca (CRIC) en el sur de Colombia. Esta organización ha logrado articular la investigación indígena y la educación intercultural de forma paradigmática. Recientemente, el CRIC ha construido una propuesta de investigación en articulación con otras dos universidades indígenas latinoamericanas, un asunto que abordaré en el siguiente apartado. Para desarrollar este análisis adopto un enfoque etnográfico, que se nutre de varias conversaciones en distintas estancias de trabajo de campo (2013, 2016 y 2018). Complemento este abordaje con el análisis documental de cartillas educativas, materiales de investigación inéditos de varios intelectuales indígenas, revisión de las revistas Çxayu'çe y Señas, ubicadas en el Centro de Documentación José María Ulcué, en la ciudad de Popayán (Colombia), y documentos de las universidades indígenas de Colombia, Ecuador y Nicaragua.

El CRIC es la primera organización indígena de Colombia y reúne a distintos pueblos, aunque el mayoritario es el pueblo nasa. Desde 1978 esta organización ha fundado sus propias escuelas y ha producido materiales pedagógicos bilingües. La educación fue concebida por el CRIC como una cuestión política: las escuelas creadas por la organización debían servir para fortalecer las recuperaciones de los territorios indígenas, los "resguardos" (Centro de Investigación y Educación Popular, 1981, pp. 93-94). El objetivo era "generar una educación para defenderse colectivamente como indígenas 
y no una educación para superarse individualmente", como recuerdan sus promotores (Programa de Educación Bilingüe Intercultural, 2004, p. 42).

Desde entonces, la definición de los currículos y la orientación de las actividades y las metodologías fueron definidas colectivamente. Cada comunidad construyó su proyecto educativo comunitario, ponderando objetivos, procedimientos y estrategias de aprendizaje. Hoy el CRIC tiene a cargo la formación de más de 16.000 estudiantes de 9 pueblos indígenas y más de 800 maestros (Bolaños, 2013, p. 179). En el 2001, el CRIC inauguró la Universidad Autónoma Indígena Intercultural (UAIIN) y dos años más tarde, un Centro Indígena de Investigaciones Interculturales, con el objetivo de formar líderes nativos capaces de colaborar con los proyectos políticos de la organización.

En los años setenta del siglo veinte, el proyecto educativo estuvo influenciado por la educación popular y por la investigación-acción participativa (Bolaños, G., comunicación personal, 16 de abril de 2016). Pero con el pasar del tiempo comenzó a primar un enfoque intercultural que se ha nutrido de la lingüística, de la etnografía y de diversas formas de colaboración indígena-académica (Ramos, A., comunicación personal, 16 de abril de 2016). En las distintas zonas donde opera el CRIC han surgido iniciativas de investigación más o menos institucionalizadas. Estas investigaciones sirven de base para la producción de las cartillas que los docentes utilizan en las escuelas (Caicedo, N., comunicación personal, 22 de febrero de 2013).

Los trabajos de investigación a los que estoy haciendo referencia comparten dos características centrales que quisiera destacar. Por una parte, se desarrollan de forma comunitaria, son procesos colectivos. Por la otra, se trata de procedimientos interculturales que combinan diversas formas de conocimiento. La escritura, el registro sonoro, la sistematización, la formulación de objetivos o la georreferenciación, son acompañados por la lectura de los sueños y las percepciones sensoriales, la consulta a los sitios sagrados, la interpretación de las nubes y de los animales, la conceptualización desde la lengua materna y el trabajo con plantas medicinales, entre otros (más detalle en Levalle, 2018). La interculturalidad aparece aquí, al mismo tiempo, como una metodología de investigación, como una característica de los equipos de trabajo - conformados por intelectuales indígenas y colaboradores externos- y como un proyecto político que postula un diálogo interétnico igualitario en el marco de una nación pluralista (Rappaport, 2008).

Gracias a estas investigaciones, los intelectuales del CRIC empezaron a pensar su proceso educativo en clave intercultural. En las cartillas educativas, el territorio adquirió mayor importancia, al igual que la figura de los médicos tradicionales (Programa de Educación Bilingüe Intercultural, s. f.). También se fortalecieron varias secuencias didácticas que incorporan recorridos por el territorio, conversaciones con los adultos mayores y rituales con los médicos tradicionales. Las cartillas escolares comenzaron a formalizar algunos 
símbolos que sintetizan la concepción territorial del pueblo nasa, como el rombo, y otros que representan la temporalidad histórica, como el espiral (Comunidad Nasa y Programa de Educación Bilingüe e Intercultural del Proyecto Nasa, 1992; Consejo Regional Indígena del Cauca, 1987).

A fuerza de movilizaciones multitudinarias, el proyecto educativo del CRIC ha conseguido el reconocimiento estatal. En 2010, el Ministerio de Educación Nacional expidió el Decreto 2500 que habilita a las organizaciones y autoridades indígenas la gestión de los centros escolares. Tres años después, con el Decreto 1953, el Estado reconoció el Sistema Educativo Indígena Propio, que rige en todo el territorio nacional, y en 2018, bajo la novel figura de "universidad pública indígena", la Universidad Autónoma Indígena Intercultural se convirtió en la primera universidad de esta clase en el país.

\section{Más allá de la investigación}

No solamente la educación oficial ejerció la violencia contra las formas de vida y los conocimientos de los pueblos indígenas. Las investigaciones que llevaron a cabo académicos de diversas disciplinas sobre estos colectivos también reprodujeron aquella violencia. James Clifford (1995) planteó que la autoridad del antropólogo se fundó sobre una premisa central que postula que los indígenas son incapaces de interpretar su realidad. Por eso, resulta más habitual encontrar investigaciones sobre que investigaciones con o de indígenas. Pero, además, como destaca Alejandro Haber (2011), los presupuestos ontológicos y epistémicos sobre los que se levanta el método científico, entre los cuales se encuentran las concepciones del tiempo y de la naturaleza que comenté antes, conducen a invisibilizar los conocimientos no modernos. Por eso, a la hora de desarrollar sus investigaciones, los miembros del CRIC debieron emprender un proceso de descolonización de la práctica de investigación (Huber, C., comunicación personal, 26 de marzo de 2016).

¿Cómo piensan la investigación estos intelectuales indígenas?, ¿qué implica investigar desde sus propias ontologías? En otro lado (Levalle, 2018) analicé este asunto para el caso del Centro Indígena de Investigaciones Interculturales de Tierradentro, una institución perteneciente a las organizaciones locales del CRIC. Ahora voy a ampliar este análisis centrándome en las reflexiones del Proyecto de Educación Bilingüe Intercultural (PEBI), una institución que el CRIC fundó en 1978 en ocasión de su V Congreso. El primer material en el que los miembros del PEBI reflexionaron sobre la investigación social es una cartilla titulada "Para qué investigamos, kih'ioia 'tkha'w ia'pa'pey" (Consejo Regional Indígena del Cauca, 1987). Aquí se intentó, por primera vez, un ejercicio explícito de descolonización de la práctica investigativa. 
En aquel material puede leerse:

"La investigación no es una actividad misteriosa realizada solamente por un grupo reducido de personas, sino que por el contrario todos investigamos en la medida que tenemos que solucionar nuestras necesidades. Así las comunidades realizamos dichos procesos cuando analizamos y discutimos nuestros programas en una asamblea, cuando nos reunimos a reflexionar sobre las experiencias obtenidas en la lucha o la producción." (Consejo Regional Indígena del Cauca, 1987, p. 14).

La investigación fue concebida como una actividad cotidiana vinculada a la vida comunitaria. Primó desde entonces una mirada con énfasis en la praxis, las investigaciones se orientan a la construcción de soluciones a los problemas colectivos: "investigamos la realidad para transformarla", afirmaban los integrantes del PEBI en 1987 (Consejo Regional Indígena del Cauca, 1987, p. 13). Los primeros trabajos abordaron las lenguas maternas, la tradición oral y las prácticas culturales y fueron desarrollados por los maestros en conjunto con los niños (Bolaños, G., comunicación personal, 16 de abril de 2016).

En la década siguiente, los procesos de investigación llevados a cabo por algunos intelectuales de la universidad del CRIC, junto a los médicos tradicionales del pueblo nasa, permitieron reconstruir la cosmovisión de este pueblo. Fue el trabajo de estos investigadores el que permitió la construcción de un relato cosmológico que hoy se plasma tanto en la tradición oral como en un conjunto de materiales escritos que alimentan los procesos escolares (Sisco, M., comunicación personal, 6 de noviembre de 2018). Para el Programa de Educación Bilingüe Intercultural (2004, p. 83) la cosmovisión es "el proceso de creación de dispositivos para analizar el mundo y actuar en él". Desde este vértice, la cosmovisión se alimenta de las vivencias y de los saberes milenarios de un pueblo, pero también recupera los hechos del presente y otras herramientas apropiadas desde otras sociedades: "Eso es lo que hoy llamamos metodología y política", concluyen.

Partiendo desde la cosmovisión nasa, los miembros de la UAIIN lograron imaginar nuevas formas de pensar la investigación. En 2008, Joanne Rappaport organizó un panel en esa casa de estudios con el objetivo de reflexionar sobre las formas indígenas de llevar a cabo la investigación (Rappaport, 2012, p. 88). En este espacio, Joaquín Viluche, un investigador del pueblo nasa, presentó una propuesta que luego enviaría al Congreso de la Asociación de Estudios Latinoamericanos (LASA) con el título "El camino de la investigación como política para recrear y revitalizar el conocimiento ancestral". El trabajo de Viluche (2010) profundiza el proceso de descolonización de la investigación, porque incorpora un conjunto de elementos de la cosmovisión nasa y los pone en juego de forma innovadora.

Aquí la investigación implica "ir tras las huellas de los mayores" y "profundizar el sentido y la interpretación del proceso cosmogónico", con lo 
cual se combina la memoria colectiva y la cosmología. Si bien el horizonte sigue siendo la autonomía indígena, el propósito de las investigaciones nasa debe ser, según Joaquín, revitalizar y recrear el "conocimiento ancestral" para "corregir los desequilibrios, para orientar, aconsejar, remediar, y vivir en equilibrio y armonía entre la comunidad y la naturaleza" (Viluche, 2010, p. 3).

$\mathrm{Al}$ igual que en el documento de los años ochenta al que me referí antes, Viluche (2010, p. 1) entiende que las comunidades siempre investigaron: "La investigación no es simplemente de los intelectuales o de los doctores, es algo muy natural en el ser humano". Las investigaciones son "mandatos de la naturaleza", esto es, una capacidad innata que "comienza desde el vientre de la madre" (Viluche, 2010, p. 3). No obstante, ahora el carácter indiferenciado de la investigación se profundiza para abarcar a todas las entidades sensibles: "Todos los que habitamos en la tierra como: los animales, las plantas, los hombres y los seres espirituales, somos investigadores de todos los días, de todas las noches para seguir siendo científicos o portadores del conocimiento ancestral" (Viluche, 2010, p. 2).

Viluche (2010, p. 4) afirma que "ya no es tiempo de realizar investigaciones superficiales, ni de hacer propuestas de temas adaptados con los esquemas occidentales" e identifica siete sentidos que, en complemento con la actividad de la pregunta, permiten desarrollar las investigaciones indígenas: la vista, el oído, el olfato, el tacto, el gusto, las señas y los sueños. ${ }^{5}$

En abril de 2016, conversé con Joaquín sobre sus ideas respecto de la investigación en el Parque Caldas, espacio central de la ciudad de Popayán.

Entonces, me explicó:

"La pregunta mía era: ¿por qué la sabiduría nuestra, a pesar de que molestaron en la historia por acabar nuestra sabiduría, no se ha acabado?, ¿qué hicieron? Resulta que la cuestión ahí está, que es natural. Y la mayor parte de la sabiduría, toda esa sabiduría sobre la luna, sobre el sol, las estrellas, toda esa sabiduría sobre el viento, sobre el fuego, sobre la tierra, sobre los animales, todas esas sabidurías permanecen gracias a la utilización de los sentidos. Y yo aprendí a investigar así porque mis abuelos me decían: "si usted quiere aprender algo, tiene que andar con la oreja parada"". (Viluche, J., comunicación personal, 1 de abril de 2016).

En las clases sobre investigación que dicta en la UAIIN, Joaquín ensaya diversas formas para enseñar estas técnicas:

5 Los médicos tradicionales del pueblo nasa sienten en su propio cuerpo los mensajes de las presencias sensibles que habitan el territorio. Las diversas sensaciones corporales mediante las cuales se expresa este lenguaje reciben el nombre de señas. Lo mismo ocurre respecto de los sueños, porque a través de ellos los médicos logran entrar en comunicación con los seres espirituales. Apelando a las señas y a los sueños, es posible predecir acontecimientos importantes (Portela Guarín, 2001). 
"J: Bueno, uno es eso. Como los estudiantes tienen que escribir, entonces escribir algo donde solamente se utilice el oído. Entonces, alguien que escribe solamente lo que olió. Que escriba sobre ese olor. Para ver qué produce. Entonces, fijate que está produciendo texto también. Produce texto pero que solamente es de escuchar. Otro texto que es solamente oler. Otro texto que es solamente de mirar. Y otro texto que será solamente de tocar.

S: ¿Cómo trabajas en el aula o en el salón o con los estudiantes esos otros sentidos como los sueños?

$\mathrm{J}$ : Ese es un poquito delicado, pero nosotros trabajamos sobre eso. Algo que la mayoría cuando van a salir, van a salir a estudiar, entonces, supongamos que soñaron con una ruana nueva. Listo. Esa ruana nueva, ¿qué significa? Ya lo empieza a utilizar. Entonces, coloco ejercicios como eso, ¿no? Bueno, si anoche soñaste con una ruana nueva, ¿qué significa? Que escriban sobre eso. Bueno, si alguien soñó con un ave en la mano, ¿qué significa? Si alguien soñó cruzando agua sucia, ¿eso qué significa? Entonces, lo que sonó empieza a escribir, a redactar, y son cosas bien interesantes que producen los estudiantes.

S: ¿Lo pueden hacer solos o necesitan la guía o la interpretación de un médico tradicional?

J: Es bueno primero hacer solo. Ya con eso van a salir una cantidad de preguntas. Y si el estudiante está interesado en eso, pues, lógicamente buscará un médico y conversará. Y ahí va aprendiendo muchas cosas." (Viluche, J., comunicación personal, 1 de abril de 2016).

A partir de 2015 las ideas de Joaquín se articularon a una propuesta que los miembros de la UAIIN comenzaron a discutir con otras dos universidades indígenas latinoamericanas: la Pluriversidad Amawtay Wasi de Ecuador, que responde a la Confederación de Nacionalidades Indígenas del Ecuador (CONAIE), y la Universidad de las Regiones Autónomas de la Costa Caribe Nicaragüense, primera universidad comunitaria intercultural de América Latina. Las tres instituciones conformaron la Red de Universidades Indígenas Interculturales y Comunitarias de Abya Yala (RUIICAY).

Este espacio de articulación tuvo como primer antecedente la Maestría en Gestión del Desarrollo con Identidad para el Buen Vivir Comunitario, que se organizó en 2008 entre las tres universidades, con el apoyo del Fondo Indígena (Palechor, 2009). Desde la RUIICAY, y con la colaboración del Instituto Internacional para la Educación Superior en América Latina y el Caribe de la Unesco, comenzó a construirse la propuesta del Cultivo y Crianza de Sabidurías y Conocimientos (CCRISAC). 
La propuesta del CCRISAC implica llevar a término el proceso de descolonización de la investigación que se venía adelantando en las experiencias previas:

"Históricamente las prácticas de investigación convencional han sido usadas para estudiar, etnografíar, registrar, conocer desde la perspectiva etnocéntrica a las diversas culturas y facilitar los procesos de colonización y homogenización del pensamiento. En consecuencia, se ha generado dependencia epistémica, desvalorizando las sabidurías de los pueblos, convirtiéndolas en mercancías y en una práctica usurpadora de las sabidurías, expresiones, conocimientos y prácticas, sin la generación de ninguna retribución para los pueblos." (Red de Universidades Indígenas Interculturales y Comunitarias de Abya Yala, 2015, p. 6).

Sobre la base de estos argumentos, los miembros de la RUIICAY decidieron reemplazar el término "investigación" por el de CCRISAC, una denominación que parte de las vivencias de los pueblos indígenas:

"Por lo anterior, se retoma la lógica de 'cultivo y crianza de sabidurías y conocimientos' como una propuesta bioética que parte de procesos colectivos desde y para los pueblos, nacionalidades y culturas, para fortalecer y revitalizar su autonomía, autodeterminación, identidad, sabidurías, expresiones, conocimientos, prácticas y episteme.” (Red de Universidades Indígenas Interculturales y Comunitarias de Abya Yala, 2015, p. 6).

Más adelante, en el documento de la RUIICAY se explica en qué consiste esta propuesta y se enumeran sus principios centrales, a saber, la relacionalidad, la comunitariedad, la reciprocidad, la complementariedad y la espiritualidad:

"Desde la perspectiva de los pueblos de Abya Yala, cultivo o cultivar expresa la relación intrínseca que mantenemos con la Madre Tierra o Pachamama. Esto significa valorar los conocimientos, expresiones, prácticas, sabidurías, las formas de cuidado y preservación de la vida. Asimismo, criar o crianza se entiende como el proceso de ayudar a buscar caminos con pertinencia cultural desde la reciprocidad, complementariedad, la comunitariedad, la promoción y preservación de principios y valores de la vida...

En consecuencia, el cultivo y [la] crianza de sabidurías y conocimientos, propicia procesos de revitalización, fortalecimiento, recreación de saberes, conocimientos, prácticas, expresiones y representaciones de los pueblos y culturas a través del diálogo intercultural e interepistémico de saberes, encuentros para la siembra, cría y cosecha de sabiduría." (Red de Universidades Indígenas Interculturales y Comunitarias de Abya Yala, 2015, p. 7). 
En la propuesta del CCRISAC, el saber es un modo de conocer que se sustenta en el sentir. Manuel Sisco, uno de los investigadores de la UAIIN que ha participado de los debates sobre el CCRISAC, defiende esta idea: los conocimientos son nociones con base racional y los saberes son aquellas nociones con base en los sentimientos (Sisco, M., comunicación personal, 6 de noviembre de 2018). Esta propuesta pretende ubicar en un plano de igualdad a los distintos caminos de reflexión que han ensayado históricamente los pueblos indígenas. ${ }^{6}$

¿Cómo se llevan a cabo los procesos de CCRISAC? En primer lugar, tal como venía haciendo la UAIIN, la investigación se sustenta en la praxis. Lo que en la tradición de investigación-acción participativa se definía bajo el concepto de "acción" (Fals Borda, 1994), ahora conforma "la cosecha", una etapa en la que se plasma la articulación entre las investigaciones y los proyectos sociales y políticos que impulsan las organizaciones. Para concluir el proceso de trabajo, el estudiante debe presentar un "emprendimiento" o una "propuesta de transformación" a realizarse en su comunidad. Los miembros de la UAIIN afirman que, de este modo, "los hallazgos o resultados retornan a la comunidad para continuar con el ciclo vital del conocimiento" (Universidad Autónoma Indígena Intercultural, 2017).

Según Manuel Sisco, esa es la particularidad de la universidad del CRIC:

"Si usted, la investigación la hace desde el enfoque CCRISAC, entonces, quiere decir que aquí sembró, va a cosechar y va a revertir la cosecha. Es la particularidad de la universidad. Porque en otras universidades simplemente usted investiga, usted se gradúa y sale, ¿cierto? Entonces, por eso, en últimas, quien aprueba si la siembra creó conocimientos y sabidurías, es la comunidad." (Sisco, M., comunicación personal, 6 de noviembre de 2018).

En segundo lugar, la participación comunitaria aparece nuevamente en primer plano. Uno de los principios del CCRISAC es la "comunitariedad", la investigación es entendida como una construcción colectiva. La "selección de la semilla", la idea y el nombre de lo que se quiere hacer deben ser discutidos en conjunto con la comunidad. También es necesario consensuar colectivamente la planeación del proceso y en el informe final se debe incluir las proyecciones, consideraciones, acuerdos y compromisos destacados del CCRISAC "elaborados conjuntamente con la comunidad" (Red de Universidades Indígenas Interculturales y Comunitarias de Abya Yala, 2015, p. 18).

6 Como muchos pueblos indígenas, los saberes se manifiestan corporalmente, por eso, Fals Borda hablaba de pueblos sentipensantes (Fals Borda, 2009). Los investigadores del pueblo nasa se han percatado de que todas las palabras que en su lengua refieren a la acción de reflexionar, se componen de la raíz ûus que se traduce como 'corazón'. Por eso, dicen, los nasa no piensan con la cabeza, sino que "corazonan" (Ramos, s. f.). 
Guillermo Santamaría, un antiguo colaborador del CRIC, me comentaba que, mediante estos procedimientos, los investigadores indígenas fueron recordando una serie de prácticas, tales como la interpretación del canto de los pájaros, el movimiento de las lechuzas, las formas de las nubes, la lectura de los sueños (Santamaría, G., comunicación personal, 20 de febrero de 2013). Entonces, se empezaron a valorar elementos que eran de su cotidianeidad, pero que habían sido invisibilizados por las ciencias occidentales. De este modo, los investigadores revitalizaron un conjunto de conocimientos que son fundamentales para establecer el equilibrio cósmico, porque son esos conocimientos los que les permiten interactuar con las presencias espirituales que conviven en el territorio.

\section{Conclusiones}

La construcción de prácticas socioeducativas alternativas en el mundo indígena no puede circunscribirse a los postulados de la educación popular. Una educación propia y autónoma requiere poner en cuestión ciertos presupuestos modernos que atentan contra las ontologías de estos pueblos, tales como la temporalidad histórica unilineal y la división entre la naturaleza y la cultura. Los procesos de investigación emprendidos por los intelectuales indígenas que analicé en este trabajo permitieron construir una educación intercultural que concibe a la diferencia como una promesa política en el camino de una nación pluralista. No obstante, para lograr este cometido, la práctica de investigación, una herencia moderna, debe también ser descolonizada.

Organizaciones de base étnica de Colombia, Educador y Nicaragua han abrevado en una concepción alternativa en el marco de la cual investigar significa cultivar y criar saberes y conocimientos comunitarios. En una aproximación profundamente praxeológica, estos trabajos se proponen recrear los conocimientos indígenas para establecer un "diálogo intercultural e interepistémico". Se trata de una experiencia de acción-reflexión orientada a transformar la realidad junto con la comunidad. Bajo este enfoque, la investigación se torna un proceso educativo en sí mismo. Pero, además, al recrear las memorias colectivas y alumbrar nuevos conocimientos, estos trabajos permiten "intercultualizar" los currículos y las prácticas educativas.

Las investigaciones indígenas continúan alumbrando nuevos caminos para la descolonización de la educación. Hoy los indígenas del CRIC plantean que la educación excede el ámbito escolar, "tiene que ser para la vida, fxi'zenxi, acompañada de la suficiente armonía y equilibrio para vivir bien en el territorio, wêt wêt fxi'zeya" (Centro Indígena de Investigaciones Interculturales de Tierradentro y Universidad Autónoma Indígena Intercultural, 2011, p. 7). De hecho, un elemento común en varias organizaciones indígenas latinoamericanas es el renovado interés por rearticular la escuela con la comunidad (Baronnet, 2009; Trapnell Forero, 2008), algo que el CRIC viene intentando desde los 
años ochenta y que ahora ha logrado acordar con el Estado en el marco del Sistema Educativo Indígena Propio.

Y también se producen nuevos debates. Si el conocimiento está en el territorio, ¿por qué pasar la mayor parte del tiempo educativo en el aula? $\mathrm{Y}$ han surgido ya varias experiencias muy interesantes que hacen eje en las prácticas agrícolas y espirituales. Si sabemos que a pesar de todos estos años de escolaridad bilingüe la lengua materna se está perdiendo, ¿por qué estudiar español?, ¿no conviene acaso retrasar la escolaridad para que el niño profundice la apropiación de su lengua? Surgen entonces nuevos problemas: ¿hasta qué punto el Estado puede aceptar experiencias educativas desescolarizadas?, ¿cómo avanzar en propuestas que hagan énfasis en lo propio sin perder de vista la articulación con los otros sectores subalternos?

El carácter "propio/popular/alternativo" de estos procesos no es simplemente aquello que remite al conocimiento cosmogónico, antes bien, en el CRIC como en el zapatismo, la educación propia es aquella que fortalece la autonomía de las comunidades en estrecha articulación con las luchas contra la violencia y la opresión (Baronnet, 2009; Bolaños, 2013). En este proceso, se vuelve necesario descolonizar la práctica de investigación para restituir la agencia y las ontologías de los pueblos indígenas. No obstante, la colaboración con los investigadores externos y la adopción de procedimientos y teorías de la ciencia occidental continúan jugando un papel clave. Una vez más se trata de volver a aquellas viejas preguntas frecuentemente olvidadas en las discusiones sobre educación escolar indígena: ¿para qué y para quién educamos?, ¿para qué y para quién investigamos?

\section{Referencias bibliográficas}

Alonso, G. y R. Díaz (eds.) (2004). Construcción de espacios interculturales. Buenos Aires: Miño y Dávila.

Baronnet, B. (2009). Autonomía y educación indigena: las escuelas zapatistas de Las Cañadas de la selva Lacandona de Chiapas [Tesis de doctorado en Ciencia Social]. México DF-París: El Colegio de México AC-Université Sorbonne Nouvelle Paris III.

Baronnet, B. (2013). Desafiando las políticas del Estado: las estrategias educativas de los pueblos originarios en Colombia y México. Pueblos y Fronteras Digital, 8, pp. 126-156.

Blaser, M. y M. de la Cadena (2009). Introducción. World Anthropologies Network (WAN). Red de Antropologías del Mundo (RAM). Electronic Journal, 4, pp. 3-10. 
Bolaños, G. (2013). La Universidad Autónoma Indígena Intercultural (UAIIN): un proceso que consolida, construye y revitaliza las culturas desde la acción organizada. ISEES, 12, pp. 87-102.

Bonfil Batalla, G.; E. E. Aguirre Beltrán; L. Arizpe y S. Gómez Tagle (1977). La declaración de Barbados II y comentarios. Nueva Antropología, II, pp. 109-125.

Briones, C.; C. Delrio; P. Lanusse; A. Szluc y A. Vivaldi (2006). Diversidad cultural e interculturalidad como construcciones sociohistóricas. En A. Amegeiras y E. Jure (eds.) (2006). Diversidad cultural e interculturalidad. Buenos Aires: Prometeo Libros y Universidad Nacional de General Sarmiento, pp. 255-264.

Brovetto, C. (2010). Educación bilingüe de frontera y políticas lingüísticas en Uruguay. Pro-Posições, 21, pp. 25-43.

Centro de Investigación y Educación Popular (1981). Consejo Regional Indígena del Cauca - CRIC. Diez años de lucha, historia y documentos. Bogotá: Controversia-CINEP.

Centro Indígena de Investigaciones Interculturales de Tierradentro, Universidad Autónoma Indígena Intercultural (2011). Programa de Desarrollo Comunitario [Documento interno]. Belalcázar: Centro Indígena de Investigaciones Interculturales de Tierradentro, Universidad Autónoma Indígena Intercultural.

Clifford, J. (1995). Dilemas de la cultura: antropología, literatura y arte en la perspectiva posmoderna. Barcelona: Gedisa.

Comisión Nacional de Trabajo y Concertación de la Educación para los Pueblos Indígenas (2009). Perfil del Sistema Educativo Indígena Propio (SEIP) [Documento interno]. Bogotá: Comisión Nacional de Trabajo y Concertación de la Educación para los Pueblos Indígenas.

Comunidad Nasa y Programa de Educación Bilingüe e Intercultural del Proyecto Nasa (1992). Kwe's 'Piyn 'i, Nuestros nombres. Popayán: CRIC.

Consejo Regional Indígena del Cauca (1987). Para qué investigamos, kih'ioia'tkha'w ia'pa'pey. Popayán: CRIC.

De la Cadena, M. (2009). Política indígena: un análisis más allá de "la política". World Anthropologies Network (WAN). Red de Antropologías del Mundo (RAM). Electronic Journal, 4, pp. 139-171. 
De Sousa Santos, B. (2010). Descolonizar el saber, reinventar el poder. Montevideo: Trilce.

Descola, P. (2012). Más allá de naturaleza y cultura. Buenos Aires: Amorrortu.

Diez, M. L. (2004). Reflexiones en torno a la interculturalidad. Revista Cuadernos de Antropología Social, 19, pp. 191-213.

Fals Borda, O. (1994). El problema de cómo investigar la realidad para transformarla por la praxis. Bogotá: Tercer Mundo.

Fals Borda, O. (2009). Una sociología sentipensante para América Latina. Bogotá: Siglo del Hombre-CLACSO.

Freire, P. (1970). Pedagogía del oprimido. Buenos Aires: Siglo XXI.

García Canclini, N. (2004). Diferentes, desiguales y desconectados. Mapas de la interculturalidad. Barcelona: Gedisa.

Haber, A. (2011). Nometodología payanesa: Notas de metodología indisciplinada. Revista de Antropología, 23, pp. 9-49.

Hale, C. (2004). El protagonismo indígena, las políticas estatales y el nuevo racismo en la época del "indio permitido". En Fundación Propaz (comp.) (2005). Paz y democracia en Guatemala: desafios pendientes. Memoria del Congreso Internacional de MINUGUA "Construyendo la paz: Guatemala desde un enfoque comparado”. Managua: Propaz-MINUGUA, pp. 51-66.

Kymlicka, W. (1996). Ciudadanía multicultural. Una teoría liberal de los derechos de las minorías. Barcelona: Paidós.

Laclau, E. (1996). Emancipación y diferencia. Buenos Aires: Ariel.

Lander, E. (2000). Ciencias sociales: saberes coloniales y eurocéntricos. En E. Lander (ed.) (2000). La colonialidad del saber: eurocentrismo y ciencias sociales. Buenos Aires: Perspectivas Latinoamericanas, pp. 4-23.

Latour, B. (1991). Nunca fuimos modernos. Ensayo de antropología simétrica. Buenos Aires: Siglo XXI.

Levalle, S. (2014). Investigación intercultural e investigación acción participativa. Un diálogo desde el suroccidente colombiano. Entramados y Perspectivas, 3, pp. 65-91.

Levalle, S. (2018). Más allá del multiculturalismo. Investigación comunitaria intercultural en el Consejo Regional Indígena del Cauca, Tierradentro, Colombia (1991-2015). Runa, 39, pp. 75-93. 
Mato, D. (2008). Diversidad cultural e interculturalidad en educación superior. Experiencias en América Latina. Caracas: IESALC-Unesco.

Mato, D. (2009). Instituciones interculturales de educación superior en América Latina. Procesos de construcción, logros, innovaciones y desafios. Caracas: IESALC-Unesco.

Organización Nacional Indígena de Colombia (2010). La lucha por la tierra, baluarte de nuestro progreso y de nuestra independencia política. Conclusiones del Primer Congreso Indígena Nacional. En E. Sánchez Gutiérrez y H. Molina Echeverri (eds.) (2010). Documentos para la historia del movimiento indígena colombiano contemporáneo. Bogotá: Biblioteca Básica de los Pueblos Indígenas de Colombia-Ministerio de Cultura, pp. 195-242.

Palechor, L. (2009). Maestría Gestión del Desarrollo con Identidad para el Buen Vivir Comunitario, 2009-2010. Çxayu'çe, 13, pp. 5-6.

Portela Guarín, H. (2001). Las señas en la cosmovisión chamánica páez. Ponencia presentada en el XXIII Congreso Internacional de Americanística, Centro Studi Americanistici Circolo Amerindiano, Perugia, Italia. Disponible en: $\quad<$ http://antropologiamedica.com/sites/default/files/2016-10/2002\%20 Las\%20senas.pdf $>$ [acceso: 9 de junio de 2020].

Primer Encuentro Continental de Pueblos Indígenas (1990). Declaración de Quito. Disponible en: <http://www.cumbrecontinentalindigena.org/quito es.php> [acceso: 9 de junio de 2020].

Programa de Educación Bilingüe Intercultural (2004). ¿Qué pasaría si la escuela...? Treinta años de construcción educativa. Popayán: Consejo Regional Indígena del Cauca.

Programa de Educación Bilingüe Intercultural (s. f.) De camino al conocimiento. Cartilla de aprestamiento. Popayán: CRIC.

Quijano, A. (2000). Colonialidad del poder, eurocentrismo y América Latina. En E. Lander (ed.) (2000). La colonialidad del saber: eurocentrismo y ciencias sociales. Buenos Aires: Perspectivas Latinoamericanas, pp. 122-151.

Ramos, I. (s. f.). "Corazonar": pensar con el corazón, saberes para sanar la madre tierra [Documento interno]. Belalcázar.

Rappaport, J. (2004). La geografía y la concepción de la historia de los nasa. En A. Surrallés y P. García Hierro (eds.) (2004). Tierradentro. Territorio indígena, percepción del entorno. Lima: Tarea Gráfica Educativa/IGWIA, pp. 173-185. 
Rappaport, J. (2008). Utopías interculturales. Intelectuales públicos, experimentos con la cultura y pluralismo étnico en Colombia. Bogotá: Universidad del Rosario.

Rappaport, J. (2012). Reflexiones sobre la investigación indígena. Señas. Revista de la Casa de Pensamiento de la Cxhab Wala Kiwe-ACIN, 2, pp. 81-94.

Rappaport, J. (2013). The challenges of indigenous research. The Challenges of Indigenous Research, 22, pp. 5-25.

Red de Universidades Indígenas Interculturales y Comunitarias de Abya Yala (2015). Cultivo y crianza de sabidurías y conocimientos. Managua: CCRISAC-URACCAN.

Rodríguez, M. E. y M. Michelena (2018). Memorias charrúas en Uruguay. Reflexiones sobre reemergencia indígena desde una investigación colaborativa. Revista sobre Acesso à Justiça e Direitos nas América, 2, pp. 180-210.

Taylor, C. (1999). El multiculturalismo y la política del reconocimiento. México DF: Fondo de Cultura Económica.

Trapnell Forero, L. (2008). Los retos de la formación docente intercultural bilingüe: la experiencia de FORMABIAP. Docencia y contextos multiculturales. Reflexiones y aportes para la formación de docentes desde un enfoque intercultural. Lima: Tarea, pp. 15-30.

Universidad Autónoma Indígena Intercultural (2017). Cultivo y crianza de saberes y conocimientos (CCRISAC) [Documento interno de trabajo colectivo]. Popayán: Universidad Autónoma Indígena Intercultural.

Vélez, C. (2008). Trayectoria de la educación intercultural bilingüe en el Ecuador. Revista Educación y Pedagogía, 20, pp. 103-112.

Viluche, J. (2010). El camino de la investigación como política para recrear y revitalizar el conocimiento ancestral. Ponencia presentada en el XXIX Congreso Internacional de la Asociación de Estudios Latinoamericanos (LASA 2010), Toronto, Canadá.

Williams, R. (1988). Marxismo y literatura. Barcelona: Península.

\section{Contribución de autoría}

Este trabajo ha sido realizado en su totalidad por Sebastián Levalle. 